\title{
The therapeutic ratio is preserved for radiotherapy or cisplatin treatment in BRCA2-mutated prostate cancers
}

\author{
Danny Vesprini, MSc, MD, FRCPC, ${ }^{*}$ Steven A Narod, MD, FRCPC,: John Trachtenberg, MD, FRCSC,; ; \\ Juanita Crook, MD, FRCPC, ${ }^{*}$ Farid Jalali, BSC,; John Preiner, MD, FRCSC; ${ }_{i}^{* *}$ Srikala Sridhar, MD, FRCPC,; \\ Robert G Bristow, MD, PhD, FRCPC* ${ }^{*}$
}

\begin{abstract}
Prostate cancers in patients with a mutation in BRCA2 have earlier disease onset and an aggressive course, often necessitating the use of systemic therapy. However, these tumours are DNA repair-defective and could respond favourably to Parp inhibitors or DNA-damaging agents, depending on the therapeutic ratio (ratio of tumour response to normal tissue toxicity). We describe 3 patients treated with precision radiotherapy or cisplatin who responded favourably to both agents, yet did not suffer undue toxicity. We review the concept of treating such patients with agents that are selectively toxic to repair-deficient tumours.
\end{abstract}

Cite as: Can Urol Assoc J 2011;5(2):e31-e35; D0l:10.5489/cuaj.10080

\section{Background}

Men with an inherited mutation in BRCA2 face an increased risk of prostate cancer ${ }^{1,2}$ and the course of their disease is often aggressive. ${ }^{3-5}$ BRCA2 is a tumour suppressor protein involved in the repair of DNA double-strand breaks via homologous recombination (HR). Tumour tissues derived from BRCA2 mutation carriers (e.g., male or female breast cancers, ovarian cancer and prostate cancer being the most common) may respond differently to radiotherapy (RT) and chemotherapy when compared to tumours with normal BRCA2 and HR protein function. In the normal tissues of BRCA1 or BRCA2 carriers, one BRCA1 or BRCA2 allele remains (heterozygote) and the normal cells maintain HR; however, there is a loss of BRCA1/2 expression in tumours from these patients and therefore functional HR is usually lost. Although the data are varied, some studies have suggested that HR-defective cell lines and xenografts can be more sensitive to ionizing radiation (IR), cisplatin, mitomycin C (MMC), etoposides, melphalan and poly(ADP-ribose) polymerase inhibitors (PARPi). ${ }^{6,7}$ These cell lines, however, have also been reported to be variably sensitive or even resistant to taxane-based chemotherapy (e.g., docetaxel)..$^{8-17}$ These data suggest that patients with a BRCA2 mutation with tumours defective in HR may be targeted by agents, such as RT, cisplatin, anthracyclines or PARPi. This is based on the concept of their inability to repair DNA double strand breaks, DNA cross links or genetic synthetic lethality, respectively. , $9,11,18$ Indeed, a recent clinical trial has shown efficacy of the PARPi named olaparib (AZD2281), in women with ovarian or breast cancer that have mutations in BRCA1/2. ${ }^{19}$ In this trial, there was report of a single prostate cancer patient with a BRCA2 mutation who responded to PARPi with a sustained $>50 \%$ reduction in PSA. To date, the clinical response of men, specifically with known BRCA2 mutations to DNA-damaging chemotherapy, has not yet been reported.

Whether normal tissues from male BRCA1/2 carriers are more sensitive to radiotherapy and chemotherapy is not known. We describe 3 patients with prostate cancer and an inherited mutation in BRCA2 who were treated with precision RT or chemotherapy (i.e., cisplatin or anthracylcines which should be more efficacious in patients with HR defective tumours). Given that patients 1 and 3 were from families with a known familial BRCA2 mutation, their mutation status was determined by direct sequencing from DNA derived from peripheral lymphocytes (Promega, Madison, WI). The mutation status of patient 2 was identified through BRCA2 screening as previously described. ${ }^{20}$ Despite our concern that these agents may cause significant acute and late toxicity in such patients with a DNA-repair deficit, none of the patients experienced any serious side effects of treatment; these patients will continue to be followed for any long-term side-effects.

\section{Patient 1 (BRCA2 mutation; (6137A)}

Patient 1 has a documented BRCA2 mutation and was diagnosed with prostate cancer with an elevated screening prostate-specific antigen (PSA) of $57 \mathrm{ng} / \mathrm{mL}$. Biopsy and staging investigations revealed a cT2a, N0, M0, and a low initial Gleason score 5 prostate adenocarcinoma. He was treated with neo-adjuvant and concurrent androgen depri- 
Vesprini et al.

vation therapy (ADT) plus 66Gy external beam RT using a $3 \mathrm{D}$ conformal technique (46Gy in 23 fractions to pelvis and prostate boost of 20Gy in 10 fractions). Despite concern of the risk of significant radiation-induced toxicity due to his BRCA2 mutation status, he experienced only mild acute genitourinary $(\mathrm{GU})$ toxicity (prospective patient-reported RTOG [radiation therapy oncology group] acute toxicity score of 2 with urinary frequency requiring the use of an alpha blocker), and no acute gastrointestinal (GI) toxicity. Biochemical failure occurred 18 months post-RT and he was started on combined androgen blockade (CAB) with bicalutamide and leuprolide. As of last follow-up (133 months post $\mathrm{RT}$ ), he has had a sustained response to $\mathrm{CAB}$, though his PSA has been trending slowly upwards. His most recent value was $1.78 \mathrm{ng} / \mathrm{mL}$ (Fig 1, part a). To date, he has experienced no late GU toxicity and minimal late Gl toxicity with a Gl RTOG score of 1 (mild intermittent rectal bleeding).

\section{Patient 2 (BRCA2 mutation; C7252T)}

Patient 2 had a BRCA2 mutation after he was diagnosed with invasive ductal carcinoma of the breast at age 58. He was treated with a modified radical mastectomy and adjuvant anthracyline-based chemotherapy (5-FU, adriamycin and cyclophosphamide [FAC]), with no evidence of recurrent disease. His BRCA2 status did not result in any excess toxicity from the anthracyclines, which introduces DNA damage that is repaired via the HR pathway. Subsequently, he was diagnosed with screen-detected prostate cancer at age 63 (initial clinical staging: cT2a NO M0, PSA $4.7 \mathrm{ng} / \mathrm{mL}$, Gleason 9). He received external beam RT to the prostate alone to a total dose of 79.8Gy in 42 fractions using intensity modulated radiotherapy (IMRT) with concurrent and adjuvant (3 years) ADT. Like patient 1 , he experienced minimal toxicity, with RTOG Grade $1 \mathrm{GU}$ toxicity (baseline nocturnal urinary frequency increased from 2 to 4 times a night) and no Gl toxicity. As of last follow-up (78 months), he reported no late radiation-induced toxicity (i.e., RTOG grade 0 for both $\mathrm{GU}$ and $\mathrm{Gl})$, and remains free of biochemical failure with a PSA of $0.90 \mathrm{ng} / \mathrm{mL}$ (Fig 1, part b). We were able to derive fibroblasts (denoted Br2DR cells) from this patient for studies of IR and MMC toxicity, as well as DNA repair studies in vitro (Fig. 2). Br2DR cells showed a slight increased sensitivity to IR and MMC based on clonogenic assays in contrast to the exquisite radiosensitivity and chemosensitivity of cells derived from patients that have defects in DNA damage sensing and repair proteins. We did observe a reduced ability for Br2Dr cells to increase the nuclear concentration of the RAD51 protein (involved in BRCA2-dependent HR repair) compared to normal fibroblasts (NDF5757). If BRCA2 function was compromised, we would have observed a complete lack of RAD51 foci at DNA breaks; we did not observe this. Therefore, the mild defects observed for the heterozygote
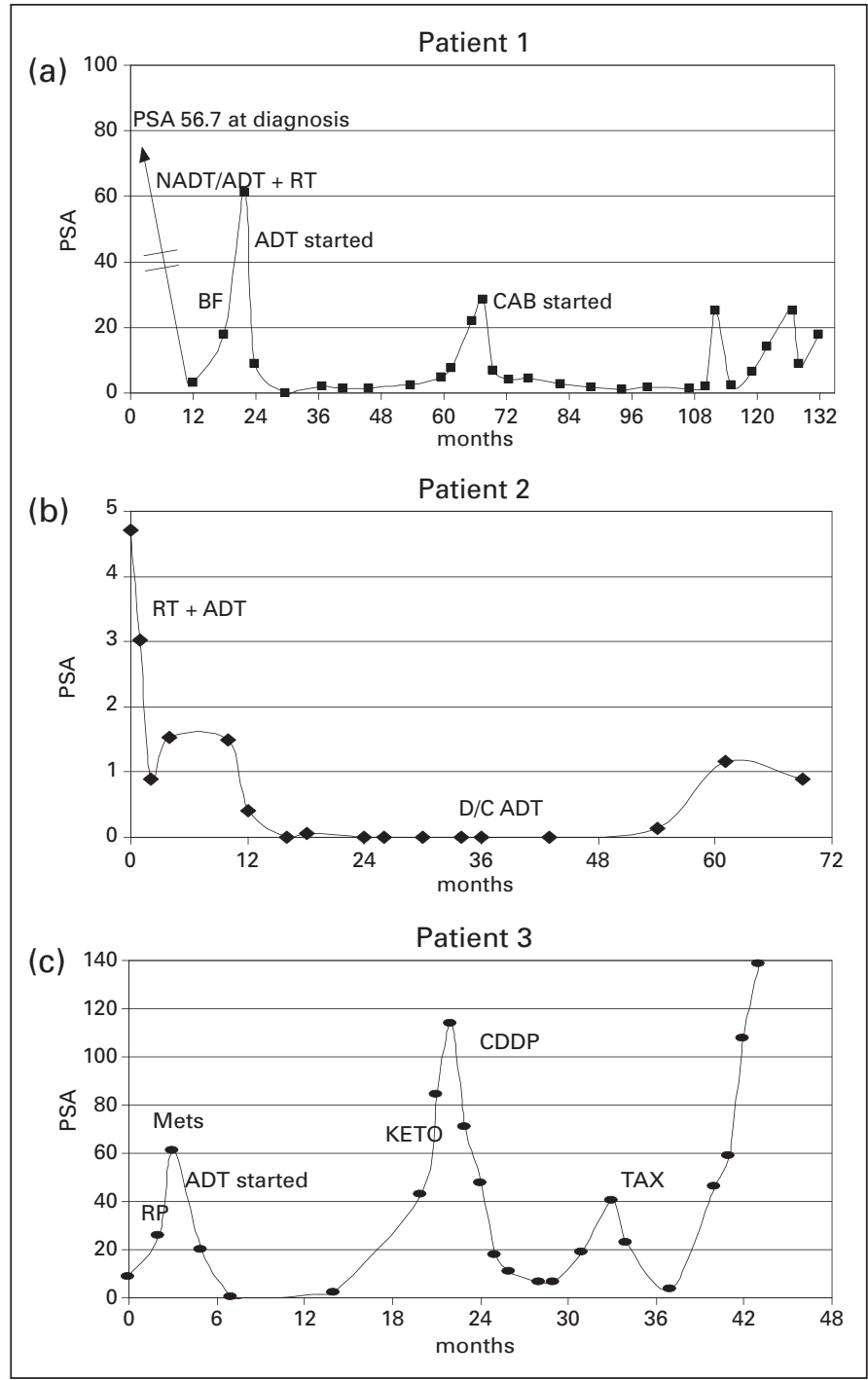

Fig. 1. Prostate-specific antigen responses to treatment in Patients 1,2 and 3 as a function of treatment and time. $A D T=$ androgen deprivation therapy. $\mathrm{BF}=$ biochemical failure. $\mathrm{CDDP}=$ cisplatin. $\mathrm{D} / \mathrm{C} \mathrm{ADT}$ = discontinuation of adjuvant androgen deprivation. KETO = ketoconozole. METS = documented metastases. NADT = neoadjuvant androgen deprivation therapy.

$\mathrm{RT}=$ Radiotherapy. $\mathrm{CAB}=$ combined androgen blockade. $\mathrm{TAX}=$ docetaxol.

Br2DR cells is consistent with previous studies, ${ }^{21-24}$ and supports our observation of minimal toxicity in this patient following precision RT.

\section{Patient 3 (BRCA2 mutation; 6503delTT)}

Patient 3 underwent a radical prostatectomy at age 49 for screen-detected intermediate risk adenocarcinoma (initial clinical staging: cT2b N0 M0, PSA 8.7, Gleason 7 [4+3]). Based on a family history of breast cancer, he was found to carry a familial BRCA2 mutation. He underwent a radical prostatectomy with final pathology showing pT3b, Gleason $9(5+4)$ adenocarcinoma, and multiple positive margins. His 


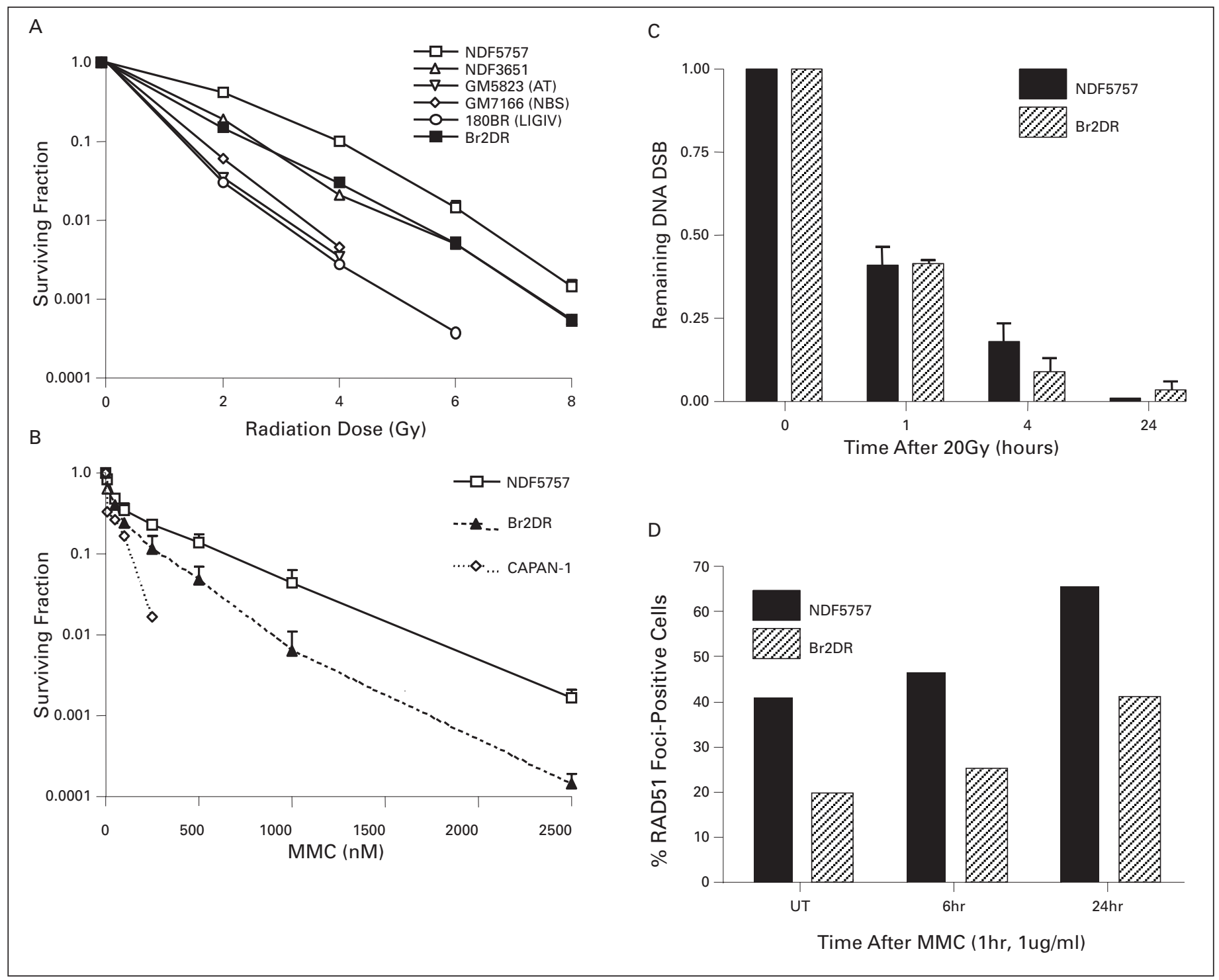

Fig. 2. DNA Repair Assays of Heterozygote Fibroblasts Derived from Patient 2 With A BRCA2 Mutation (a,b) Shown are clonogenic survival of fibroblasts derived from patient 2 ( $B r 2 D R$ strain) relative to normal fibroblasts or fibroblasts defective in DNA damage sensing or repair. In (a), Br2DR cells are similar in sensitivity following ionizing radiation relative to unaffected normal fibroblast strains (NDF5757 and 3651 strains). Note exquisite sensitivity in cell strains derived from DNA damage sensing- or repair-deficient cells derived from patients with abnormal ATM, NBS1 and DNA ligase IV proteins. In (b), Br2DR cells show increased sensitivity to Mitomycin C (MMC) ( $<<0.05$ for each dose point) relative to a normal NDF5757 strain. Also shown in (b) is the BRCA2-deficient CAPAN-1 cell line for comparison showing exquisite MMC sensitivity. (c) Results from the neutral Comet assay measuring residual DNA double strand breaks (DSBs) as a function of time (0-24 hours) following a dose of $20 \mathrm{~Gy}$. Br2DR cells show a non-significant trend towards increased residual DSBs at 24 hours. (d) Data from a RAD51 protein nuclear foci assay in which the ability for cells to increase the nuclear fraction of RAD51 foci (required for homologous recombination pathway of DSb repair) at sites of DNA damage is measured using fluorescent microscopy. An example of the foci scored in this technique is shown in the inset. The data are presented at the percent of cells positive for RAD51 foci before and after treatment with the DNA cross-linking agent, mitomycin C (MMC). A decreased RAD51 response in the Br2DR cells is observed compared to normal fibroblasts suggesting a decreased ability to mobilize RAD51 to the nucleus. Note: cells completely deficient in BRCA1 and BRCA2 function have no ability to up-regulate RAD51 foci at all.

postoperative PSA never reached an undetectable level, and at 3 months post-surgery rose to $26 \mathrm{ng} / \mathrm{mL}$. Staging revealed multiple bone metastases, and he was started on $\mathrm{CAB}$ and his PSA declined to $0.47 \mathrm{ng} / \mathrm{mL}$. By 18 months, however, he had castrate-resistant disease with PSA rising to $43 \mathrm{ng} / \mathrm{mL}$ and pelvic and abdominal lymph adenopathy and multiple bone metastases demonstrated with a computed tomography scan (Fig 1, part c). He was treated with ketoconozole and prednisone with no biochemical response. Due to a PSA doubling time of less than 2 months and increasing lesions on his bone scan, he was started on chemotherapy. Although standard treatment would be docetaxel, due to his BRCA2 status, he was started on cisplatin, monthly at $100 \mathrm{mg} / \mathrm{m}^{2}$. He tolerated this treatment very well, with only mild toxicity 
Vesprini et al.

and responded well with a drop in his PSA from $114 \mathrm{ng} / \mathrm{mL}$ pre-chemotherapy to $6.5 \mathrm{ng} / \mathrm{mL}$ after 8 cycles of cisplatin. He also experienced an overall improvement in his quality of life, except for moderate, but increasing, peripheral neuropathy. Within 2 months, his PSA again rapidly doubled and symptomatically he felt worse with increasing bone pain. He then received 3 cycles of docetaxel with evidence of both PSA and symptomatic responses. Although his PSA declined from $40.39 \mathrm{ng} / \mathrm{mL}$ pre-chemotherapy to $3.6 \mathrm{ng} / \mathrm{mL}$ with 3 cycles of docetaxol, further treatment was discontinued due to fatigue, nausea and dehydration. He was well for 2 months, but again his PSA doubled within another 2 months and he experienced increasing back pain; he is being considered for alternate systemic regimens.

\section{Discussion}

To our knowledge, this is the first report of tumour and normal tissue responses in BRCA2-associated prostate cancer following RT or chemotherapy regimens that directly target DNA. Whether these patients can be routinely approached with DNA-damaging agents that take advantage of HR-deficient tumours without significant additional toxicity requires defined and multi-institutional clinical trials in these rare cohorts. Given the results of the recent phase 1 study with olaparib, ${ }^{19}$ optimal treatment in patients whose PSA is rising following definitive local therapy (e.g., radical prostatectomy or RT) could one day involve first-line salvage treatment with PARPi if it is shown to be more efficacious than taxanes or mitoxantrone within clinical trials. If shown to be efficacious, PARPi could be preferable to the use of long-term ADT given the recent concerns of diabetic and cardiac complications in older men receiving hormone therapy. ${ }^{25,26}$ We suggest that long-term studies of PARPi and other HR-targeting agents should be completed to compare their relative utility to existing treatment strategies. ${ }^{10,14}$

Studies to date estimate that between $0.8 \%$ and $2.0 \%$ of men with prostate cancer have an inherited mutation in BRCA2 ${ }^{27,28}$ In Toronto, we see about 3000 new prostate cancer patients a year, which translates into potentially 30 to 60 BRCA2-positive cases per year. Two studies have reported that survival in BRCA2-associated prostate cancers is poor. ${ }^{4,5}$ BRCA2 patients appeared to have a worse prognosis than those with no mutation ${ }^{5}$ or with a BRCA1 mutation (median survival of 4 years for BRCA2 vs. 8 years for BRCA1). ${ }^{4}$ It is therefore important to consider that these patients may not always benefit from the sole use of local treatment with surgery or RT and may require adjuvant systemic therapy to increase survival.

The BRCA2 carrier status of patient 3 was known shortly after his diagnosis and, on the basis of his genotype, we elected to initially treat him with cisplatin, rather than a taxane (in which the toxicity mechanism is independent of DNA repair). After developing castrate-resistant prostate cancer, this 49-year-old patient showed a good initial response to platinum therapy. A similar approach has been used in BRCA1-associated breast cancer, with encouraging results. ${ }^{29}$ Surprisingly, despite in vitro and in vivo preclinical data suggesting that HR defective cancer cells are resistant to taxane-based treatment, ${ }^{12}$ patient 3 also responded to docetaxel with a greater than $50 \%$ reduction in PSA. Further data are required to understand the relative response to different chemotherapy regimens in HR-defective tumours. At least one study has suggested that BRCA2 expression may predict response to doxetaxel in breast cancer. ${ }^{30}$ As such, we suggest that patients 1 and 2 remain candidates for treatment with cisplatin, taxanes or PARPi in case they develop castrate-resistant disease.

We did not observe any excess toxicity following precision RT or chemotherapy using cross-linking agents; all DNA-damaging treatments theoretically lead to toxicity in HR-deficient patients. This supports selected in vitro and in vivo pre-clinical data which shows that heterozygosity for BRCA1 or BRCA2 in a carrier's normal tissues does not manifest as an increase in toxicity due to intrinsic HR deficiency. ${ }^{9}$ Furthermore, there is no evidence to date that breast cancer patients with a BRCA1 or BRCA2 mutation have increased acute or late toxicity to radiation or chemotherapy, ${ }^{31}$ but follow-up studies on these patients are ongoing.

\section{Conclusion}

The 3 patients with BRCA2 mutations in this report had cancers with aggressive features which we believe benefited from systemic therapy in a combined modality setting. Cisplatin is currently being evaluated in women with breast cancer and a BRCA1 mutation with encouraging early results. ${ }^{29}$ It may be that BRCA2 carriers with prostate cancer would benefit most from combined modality therapy, based on the synthetic lethality approach, with cisplatin and/or PARPi and local-regional RT and ADT. We believe that clinical trials with agents such as PARPi and/or cisplatin provide a unique opportunity to test the hypothesis that DNA repairdeficient cancers will have genetically-defined sensitivity. However, these trials need to be carefully completed and compared with taxane-based therapies to demonstrate additional efficacy without undue toxicity in men with relative aggressive, BRCA2-associated prostate cancers. Information regarding toxicity and response to therapy is important to help guide management in these unique and rare patients whose genetic background may characterize the clinical course and treatment of their disease.

*Department of Radiation Oncology, University of Toronto, Toronto, ON; 'Sunnybrook Odette Cancer Centre, Toronto, ON; \pm Women's College Research Institute, University of Toronto, Toronto, ON; ¿Division of Urology, Department of Surgery, University of Toronto, Toronto, ON; *Ontario Cancer 
Institute/Princess Margaret Hospital (University Health Network), Toronto, ON; ** Southlake Regional Cancer Centre, Newmarket, ON; ${ }^{+\dagger}$ Department of Medical Oncology and Hematology, University of Toronto, Toronto, ON

Acknowledgements: The authors would like to thank Kirsten Currie, Sheri Horsburgh and Andrew Matthew for their helpful comments. This work is supported by an Ontario Institute for Cancer Research operating grant to RGB and a group grant from Prostate Cancer Canada. This study was reviewed by the PMH-UHN Ethics Board and approved as REB \# 10-0619-CE.

Competing interests: None declared.

This paper has been peer-reviewed.

\section{References}

1. The Breast Cancer Linkage Consortium. Cancer risks in BRCA2 mutation carriers. The Breast Cancer Linkage Consortium. J Natl Cancer Inst 1999:91:1310-6.

2. Thompson D, Easton DF. Cancer Incidence in BRCA1 mutation carriers. J Natl Cancer Inst 2002;94:135865.

3. Mitra A, Fisher C, Foster $C S$, et al. Prostate cancer in male BRCA1 and BRCA2 mutation carriers has a more aggressive phenotype. Br J Cancer 2008;98:502-7.

4. Narod SA, Neuhausen S, Vichodez G, et al. Rapid progression of prostate cancer in men with a BRCA2 mutation. Br J Cancer 2008;99:371-4.

5. Tryggvadottir L, Vidarsdottir L, Thorgeirsson T, et al. Prostate cancer progression and survival in BRCA2 mutation carriers. J Natl Cancer Inst 2007;99:929-35.

6. Higgins $G S$, Prevo R, Lee YF, et al. A small interfering RNA screen of genes involved in DNA repair identifies tumour-specific radiosensitization by POLQ knockdown. Cancer Res 2010;70:2984-93.

7. Emestos B, Nikolaos $P$, Koulis $G$, et al. Increased chromosomal radiosensitivity in women carrying BRCAI/ BRCA2 mutations assessed with the 62 assay. Int I Radiat Oncol Biol Phys 2010;76:1199-205.

8. Ashworth A. A synthetic lethal therapeutic approach: poly(ADP) ribose polymerase inhibitors for the treatment of cancers deficient in DNA double-strand break repair. I Clin Oncol 2008;26:3785-90.

9. Bristow RG, Ozcelik H, Jalali F, et al. Homologous recombination and prostate cancer: a model for novel DNA repair targets and therapies. Radiother Oncol 2007;83:220-30.

10. Evers B, Schut E, van der Burg E, et al. A high-throughput pharmaceutical screen identifies compounds with specific toxicity against BRCA2-deficient tumours. Clin Cancer Res 2010;16:99-108.

11. Helleday T, Petermann E, Lundin C, et al. DNA repair pathways as targets for cancer therapy. Nat Rev Cancer 2008;8:193-204.

12. Powell SN, Kachnic LA. Therapeutic exploitation of tumour cell defects in homologous recombination. Anticancer Agents Med Chem 2008;8:448-60.

13. Promkan $M$, Liv $G$, Patmasiriwat $P$, et al. BRCAl modulates malignant cell behavior, the expression of survivin and chemosensitivity in human breast cancer cells. Int J Cancer 2009; 125:2820-8.
14. Sandhu SK, Yap TA, de Bono IS. Poly(ADP-ribose) polymerase inhibitors in cancer treatment: a clinical perspective. Eur J Cancer 2010;46:9-20.

15. Tassone P, Di Martino MT, Ventura M, et al. Loss of BRCAl function increases the antitumour activity of cisplatin against human breast cancer xenografts in vivo. Cancer Biol Ther 2009;8:648-53.

16. Tassone P, Tagliaferri P, Perricelli A, et al. BRCAl expression modulates chemosensitivity of BRCA1-defective HCC1937 human breast cancer cells. Br J Cancer 2003:88:1285-91.

17. Treszezamsky AD, Kachnic LA, Feng Z, et al. BRCAT-and BRCA2-deficient cells are sensitive to etoposideinduced DNA double-strand breaks via topoisomerase II. Cancer Res 2007;67:7078-81.

18. Fourquet A, Stoppa-Lyonnet D, Kirova YM, et al. Familial breast cancer: clinical response to induction chemotherapy or radiotherapy related to BRCAl /2 mutations status. Am I Clin Oncol 2009;32:127-31.

19. Fong PC, Boss DS, Yap TA, et al. Inhibition of poly(ADP-ribose) polymerase in tumours from BRCA mutation carriers. N Engl J Med 2009;361:123-34.

20. Risch HA, McLaughlin JR, Cole DE, et al. Prevalence and penetrance of germline BRCA1 and BRCA2 mutations in a population series of 649 women with ovarian cancer. Am J Hum Genet 2001;68:700-10.

21. Morimatsu M, Donoho G, Hasty P. Cells deleted for Brca2 $\mathrm{COOH}$ terminus exhibit hypersensitivity to gamma-radiation and premature senescence. Cancer Res 1998;58:3441-7.

22. Nieuwenhuis B, Van Assen-Bolt AJ, Van Waarde-Verhagen MA, et al. BRCA1 and BRCA2 heterozygosity and repair of X-ray-induced DNA damage. IntJ Radiat Biol 2002;78:285-95.

23. Sharan SK, Morimatsu M, Albrecht U, et al. Embryonic lethality and radiation hypersensitivity mediated by Rad51 in mice lacking Brca2. Nature 1997;386:804-10.

24. Tutt A, Connor F, Bertwistle D, et al. Cell cycle and genetic background dependence of the effect of loss of BRCA2 on ionizing radiation sensitivity. Oncogene 2003;22:2926-31.

25. Keating NL, $\mathrm{O}^{\prime}$ Malley AJ, Freedland SJ, et al. Diabetes and cardiovascular disease during androgen deprivation therapy: observational study of veterans with prostate cancer. J Natt Cancer Inst 2010;102:39-46.

26. Levine GN, D'Amico AV, Berger $P$, et al. Androgen-deprivation therapy in prostate cancer and cardiovascular risk: a science advisory from the American Heart Association, American Cancer Society, and American Urological Association: endorsed by the American Society for Radiation Oncology. Circulation 2010;121:833-40.

27. Gayther SA, de Foy KA, Harrington $P$, et al. The frequency of germ-line mutations in the breast cancer predisposition genes BRCA1 and BRCA2 in familial prostate cancer. The Cancer Research Campaign/British Prostate Group United Kingdom Familial Prostate Cancer Study Collaborators. Cancer Res 2000;60:4513-8.

28. Ostrander EA, Udler MS. The role of the BRCA2 gene in susceptibility to prostate cancer revisited. Cancer Epidemiol Biomarkers Prev 2008; 17:1843-8.

29. Byrski T, Huzarski T, Dent R, et al. Response to neoadjuvant therapy with cisplatin in BRCAl-positive breast cancer patients. Breast Cancer Res Treat 2009;115:359-63

30. Egawa C, Miyoshi Y, Takamura Y, et al. Decreased expression of BRCA2 mRNA predicts favorable response to docetaxel in breast cancer. Int J Cancer 2001;95:255-9.

31. Pierce $L$, Strawderman $M$, Narod $S A$, et al. Effect of radiotherapy after breast-conserving treatment in women with breast cancer and germline BRCAl/2 mutations. J Clin Oncol 2000;18:3360-9.

32. Al Rashid ST, Dellaire G, Cuddihy A, et al. Evidence for the direct binding of phosphorylated p53 to sites of DNA breaks in vivo. Cancer Res 2005;65:10810-21.

33. Bhogal $\mathrm{N}$, Kaspler $\mathrm{P}$, Jalali $\mathrm{F}$, et al. Late residual gamma-H2AX foci in murine skin are dose responsive and predict radiosensitivity in vivo. Radiat Res 2010;173:1-9

34. Choudhury A, Zhao H, Jalali F, et al. Targeting homologous recombination using imatinib results in enhanced tumour cell chemosensitivity and radiosensitivity. Mol Cancer Ther 2009:8:203-13.

Correspondence: Dr. Robert G Bristow, Princess Margaret Hospital, 610 University Avenue, Toronto, Ontario, Canada M4X 1E5; tel: 416-946-2936; fax: 416-946-4586; rob.bristow@rmp.uhn.on.ca 